\title{
Correction: Tham, P.E., et al. Recovery of Protein from Dairy Milk Waste Product Using Alcohol-Salt Liquid Biphasic Flotation. Processes 2019, 7, 875
}

\author{
Pei En Tham ${ }^{1}$, Yan Jer Ng ${ }^{1}$, Revathy Sankaran ${ }^{2}{ }^{\oplus}$, Kuan Shiong Khoo ${ }^{1}$, Kit Wayne Chew ${ }^{3, *}$, \\ Yee Jiun Yap ${ }^{3}$, Masnindah Malahubban ${ }^{4}$, Fitri Abdul Aziz Zakry ${ }^{4}$ and Pau Loke Show ${ }^{1, *(1)}$ \\ 1 Department of Chemical and Environmental Engineering, Faculty of Science and Engineering, \\ University of Nottingham Malaysia, Jalan Broga, Semenyih 43500, Selangor Darul Ehsan, Malaysia; \\ peien0405@gmail.com (P.E.T.); yanjer98@hotmail.com (Y.J.N.); kuanshiong.khoo@hotmail.com (K.S.K.) \\ 2 Institute of Biological Sciences, Faculty of Science, University of Malaya, Jalan Universiti, \\ Kuala Lumpur 50603, Malaysia; revathysankaran@ymail.com \\ 3 School of Mathematical Sciences, Faculty of Science and Engineering, University of Nottingham Malaysia, \\ Jalan Broga, Semenyih 43500, Selangor Darul Ehsan, Malaysia; YeeJiun.Yap@nottingham.edu.my \\ 4 Faculty of Agriculture and Food Sciences, Universiti Putra Malaysia Sarawak Campus, \\ Bintulu 97008, Sarawak, Malaysia; masnindah@upm.edu.my (M.M.); zakryfitri@upm.edu.my (F.A.A.Z.) \\ * Correspondence: KitWayne.Chew@nottingham.edu.my or kitwayne.chew@gmail.com (K.W.C.); \\ PauLoke.Show@nottingham.edu.my or showpauloke@gmail.com (P.L.S.); Tel.: +60-3-8924-8605 (P.L.S.)
}

We were not aware of some errors made in the proofreading phase; therefore, we wish to make the following corrections to the mathematical equations in the text.

(1) The bar at some units are placed on the bottom instead of the top, the correct representation for the following equations should be: Flux $\bar{J}$ is defined as the number of bubbles passing though an area $A$ per unit time. Assuming no diffusion, i.e., only convection,

$$
\bar{J}=\sigma \bar{S}
$$

where $\sigma$ and $\bar{s}$ are the density and velocity of the bubbles respectively. Putting Equation (2) into (1) gives

$$
\frac{\partial \sigma}{\partial t}=-\nabla \cdot \bar{J}
$$

The bubbles are assumed to move into the region $\mathrm{R}$, resulting in a negative $\nabla \cdot \bar{J}$, and the negative sign is to make it positive. To solve Equation (16), Laplace transform is applied to Equation (16), giving

$$
\begin{gathered}
f(z) \bar{\sigma}_{z}(z, s)+s \bar{\sigma}(z, s)-\sigma(z, 0)+F(z) \bar{\sigma}(z, s)=0 \\
f(z) \bar{\sigma}_{z}(z, s)+[s+F(z)] \bar{\sigma}(z, s)-\sigma(z, 0)=0
\end{gathered}
$$

Since $\sigma(z, 0)=0$, Equation (17) becomes

$$
f(z) \bar{\sigma}_{z}(z, s)+[s+F(z)] \bar{\sigma}(z, s)=0
$$

To solve Equation (18), we divide Equation (18) by $f(z)$ to give

$$
\bar{\sigma}_{z}(z, s)+\frac{s+F(z)}{f(z)} \bar{\sigma}(z, s)=0
$$


Multiplying Equation (19) with integrating factor $e^{\int \frac{s+F(z)}{f(z)} d z}$ yields

$$
\frac{d}{d z}\left[e^{q(z, s)} \bar{\sigma}(z, s)\right]=0
$$

Integrating Equation (20) gives

$$
\bar{\sigma}_{z}(z, s)=C e^{Q(z, s)}
$$

(2) The cylindrical coordinates $\varnothing$ should have the terms as followed: $\varnothing=\tan ^{-1} \frac{y}{x}$.

(3) There is an additional ' $1 \mathrm{n}$ ' term in both the equations which should not be present. The correct equations are as shown below:

$$
q(z, s)=\frac{9 s v}{8 \rho g \alpha^{2}}\left(\frac{\rho^{2} g^{2} z^{3}}{3}-\rho g z^{2} P_{e x}+\left(P_{e x}\right)^{2} z\right)+\ln \left|\frac{8 \rho g \alpha^{2}}{9 v\left(\rho g z-P_{e x}\right)^{2}}\right|
$$

Transforming Equation (22) from the $s$ domain back to the $t$ domain yields

$$
L^{-1}\left\{\bar{\sigma}_{z}(z, s)\right\}=\sigma_{z}(z, t)=\delta\left[t-\frac{9 v}{8 \rho g \alpha^{2}}\left(\rho g z^{2} P_{e x}-\left(P_{e x}\right)^{2} z-\frac{\rho^{2} g^{2} z^{3}}{3}\right)-\ln \left|\frac{8 \rho g \alpha^{2}}{9 v\left(\rho g z-P_{e x}\right)^{2}}\right|\right]
$$

where $L$ is the Laplace transform.

\section{Reference}

1. Tham, P.E.; Ng, Y.J.; Sankaran, R.; Khoo, K.S.; Chew, K.W.; Yap, Y.J.; Malahubban, M.; Aziz Zakry, F.A.; Show, P.L. Recovery of Protein from Dairy Milk Waste Product Using Alcohol-Salt Liquid Biphasic Flotation. Processes 2019, 7, 875. [CrossRef]

(C) 2020 by the authors. Licensee MDPI, Basel, Switzerland. This article is an open access article distributed under the terms and conditions of the Creative Commons Attribution (CC BY) license (http://creativecommons.org/licenses/by/4.0/). 